\title{
De la pregunta por el ser al ser de la pregunta. Dos malentendidos en torno a Deleuze y Heidegger
}

Pablo Pachilla

Consejo Nacional de Investigaciones Científicas y Técnicas, Argentina.

Recibido el 02/05/2020. Aceptado el 05/07/2020.

\begin{abstract}
Resumen
El presente trabajo se propone despejar dos malentendidos habituales en la comparación entre el pensamiento de Gilles Deleuze y el de Martin Heidegger: en primer lugar, la supuesta oposición entre la negatividad heideggeriana y la positividad deleuziana; en segundo lugar, la contrariedad presunta entre la inmanencia deleuziana y la trascendencia heideggeriana. Se propone de este modo no tanto señalar coincidencias como subrayar que las diferencias no están allí donde se las suele buscar. El método utilizado consiste en tomar como hilo conductor las menciones a Heidegger en Diferencia y repetición (1968) para rastrear a partir de allí el uso que Deleuze hace de conceptos heideggerianos. Intentaremos mostrar que los dos aspectos del campo trascendental deleuziano, lo virtual y lo intensivo, revisten una cierta "negatividad" y un "ocultamiento", respectivamente, lo cual se contrapone a la imagen de Deleuze como un filósofo del darse pleno sin sustracción. Argumentaremos asimismo que la trascendencia heideggeriana no necesariamente se opone a la inmanencia deleuziana, y que los conceptos heideggerianos de Zwiefalt y Selbe son utilizados por Deleuze para pensar la inmanencia. Sostenemos que la distinción entre lo negativo y lo problemático le permite a Deleuze hablar del ser aun aceptando la diferencia ontológica.
\end{abstract}

Palabras clave: Deleuze, Heidegger, negatividad, pliegue, trascendencia

\section{From the Question Concerning Being to the Being of the Question. Two Misunderstandings around Deleuze and Heidegger}

\begin{abstract}
This paper aims to clear two regular misunderstandings when comparing the thoughts of Gilles Deleuze and Martin Heidegger: firstly, the alleged opposition between Heideggerian negativity and Deleuzian positivity; secondly, the assumed contrariety between Deleuzian immanence and Heideggerian transcendence. In this fashion, the proposal is not so much to point at coincidences as to underline the fact that the differences are not where they are usually sought. The method used consists in taking Deleuze's mentions to Heidegger in Difference and Repetition (1968) as a common thread for
\end{abstract}


tracking the Deleuzian use of Heideggerian concepts. We will attempt to show that both aspects of the Deleuzian transcendental field, the virtual and the intensive, hold a certain "negativity" and "concealment", respectively, which runs against the picture of Deleuze as a philosopher of thorough and unconcealed manifestation. We will likewise argue that Heideggerian transcendence is not necessarily opposed to Deleuzian immanence, and that the Heideggerian concepts of Zwiefalt and Selbe are used by Deleuze in order to think of immanence. We contend that the distinction between the negative and the problematic allows Deleuze to speak of being even while accepting ontological difference.

Keywords: Deleuze, Heidegger, fold, negativity, transcendence

\section{Introducción}

Deleuze, pensador de la inmanencia; Heidegger, de la trascendencia. Deleuze, filósofo de la positividad; Heidegger, de la negatividad. Se trata de prejuicios muy difundidos que encuentran su origen en la vaguedad con la que se piensan a veces dichos conceptos, los cuales no significan nada a menos que se los determine al interior del pensamiento de cada autor. Tarea de largo aliento, con respecto a la cual aquí solo se harán algunas indicaciones. No pretendemos desde luego asimilar a dos filósofos cuyos estilos, conceptos y vocabulario difieren sobremanera en una superposición que desdibuje a ambos. Sin embargo, ignorar la influencia de Heidegger sobre Deleuze, así como los ecos y disonancias entre ambos pensamientos, sería hacer la vista gorda de toda una constelación problemática en la cual Deleuze, en tanto filósofo escribiendo en Francia en los años '60, se encontraba necesariamente inserto.

No es el objetivo de este trabajo realizar un análisis histórico de las apariciones de la figura de Heidegger a lo largo de la vida filosófica de Deleuze, lo cual nos llevaría, entre otras cosas, a discutir más detalladamente la noción de pliegue tal como aparece en su obra tardía. No obstante, resulta de utilidad hacer unas breves menciones para tener un panorama que dé cuenta del origen de la "rivalidad" con Heidegger tan dada por sentado. El encuentro de Deleuze con Heidegger se remonta a su época de estudiante de colegio secundario en liceo Henri IV, donde Jean Beaufret, el máximo exponente del heideggerianismo en Francia - y destinatario de la famosa "Carta sobre el humanismo" del pensador oriundo de Messkirch-, exclamó que para entender a Heidegger era preciso hablar y pensar en alemán. A la semana siguiente, Deleuze intervino en la clase para decir que el escritor francés Alfred Jarry -creador de la patafísica - no solo había entendido sino que había precedido a Heidegger (cf. Dosse, 2009: 128). Beaufret le habría pedido a Deleuze que se callara, pero esta idea dio lugar a dos textos publicados años después (Deleuze 1992; 2002). Las humoradas deleuzianas con respecto al filósofo alemán no terminan ahí: en el número que Magazine littéraire dedicó a Deleuze, aparece una breve nota biográfica redactada por el propio filósofo que reza: "Viaja poco, no adhirió jamás al Partido Comunista, no fue nunca fenomenólogo ni heideggeriano, no renunció nunca a Marx, no repudió Mayo del '68" (Deleuze, 1988b). Por último, cabe mencionar que, tal como destaca Dosse, revitalizar a Hume en un contexto intelectual dominado por "las tres haches" (Hegel, Husserl, Heidegger) requiere "un arte consumado del contrapunto" (Dosse, 2009: 144; cf. Deleuze, 1953).

1 Para un análisis exhaustivo de esta noción, cf. van Tuinen y McDonnell, 2010.

2 Salvo que se indique lo contrario, todas las traducciones son de nuestra autoría. En cuanto a la obra de Heidegger, abreviamos GA por Gesamtausgabe para indicar el original alemán, seguido del número de tomo y la página. 
A pesar de ello, la presencia de Heidegger en el magnum opus deleuziano, Diferencia y repetición (1968), no es nada desdeñable. El índice de nombres y de temas que se encuentra al final de la obra tiene tres columnas: autor, obra y páginas, temas. Heidegger aparece allí, pero en obras encontramos "passim"; en cuanto a los temas, leemos "La diferencia ontológica (Ser, diferencia, pregunta)" (1993: 397). La ausencia de una obra encuentra una explicación en la presencia una obra, lo cual se encuentra explicitado por Deleuze en las palabras previas al listado. En efecto, Deleuze recalca allí que toma la obra de Heidegger y no solo esta o aquella obra: "A veces invocamos de manera alusiva, vaga y general autores $u$ obras sin embargo esenciales: por ejemplo, Damascio, Schelling, Heidegger para la filosofía de la diferencia" (391). Y unas líneas más abajo: "Para ciertos autores (Platón, Aristóteles, Leibniz, Hegel, Freud, Heidegger) hemos simplemente indicado passim en la columna de las obras. Es que los temas de la diferencia o de la repetición están realmente presentes en el conjunto de su obra" (391). Nótese la compañía del filósofo alemán, en un listado que comienza con Platón y termina con él. Si a ello le sumamos su aparición nada menos que en la primera página del libro, se vuelve innegable que la influencia de Heidegger en la principal obra de ontología deleuziana es de primera importancia. ${ }^{3}$ Escribe allí Deleuze que el tema del libro está en el aire de los tiempos, algunos de cuyos signos comienza a relevar: "la orientación cada vez más acentuada de Heidegger hacia una filosofía de la Diferencia ontológica" es ni más ni menos que el primero de estos signos (1993: 1).

Más allá de dicha ubicuidad, Deleuze menciona a lo largo del libro diversos textos tanto de como sobre Heidegger: Sery tiempo, "¿Qué es metafísica?", "De la esencia del fundamento" (en Hitos [Wegmarken]), "Superación de la metafísica", "¿Qué significa pensar?", "...Poéticamente habita el hombre..." (en Conferencias y artículos [Vorträge und Aufsätze]), Identidad y diferencia, Kant y el problema de la metafísica, en cuanto a los primeros; Hölderlin y Heidegger de Beda Allemann, El juego como símbolo del mundo de Eugen Fink, Hacia el pensamiento planetario de Kostas Axelos y la introducción de Jean Beaufret al Poema de Parménides en cuanto a los segundos. Puesto que dar cuenta de todas las menciones y, más aún, hacer justicia a todos los problemas y conceptos allí involucrados, requeriría un trabajo de otras dimensiones, nos proponemos aquí simplemente aclarar dos supuestas oposiciones que funcionan como lugares comunes que impiden poner en relación a estos dos autores: en primer lugar, aquella entre la trascendencia heideggeriana y la inmanencia deleuziana; en segundo lugar, aquella entre la negatividad heideggeriana y la positividad deleuziana. Para ello - si bien nuestro corpus no se limitará a ello-, nuestro método consistirá en tomar como hilo conductor la puesta en relación entre las nociones ontológicas principales de Diferencia y repetición y algunas de las menciones a Heidegger presentes en dicho libro.

\section{Negatividad y positividad}

\subsection{Negatividad}

El primer capítulo de Diferencia y repetición termina -justo antes de la recapitulación final - con una muy extensa nota al pie intitulada "Nota sobre la filosofía de la diferencia de Heidegger" (1993: 89-91). Deleuze viene de explorar el estatuto de la negatividad específica de la Diferencia virtual, que no es una negación cualquiera; esta última, por el contrario, no es sino la sombra de la afirmación cuestionante de la diferencia. 
Deleuze propone las expresiones "(no)-ser" y "?-ser" (1993: 89) para referirse a ese estatuto no-empírico de la Diferencia, que no es nada actual y en ese sentido porta una cierta negatividad, pero es crucial no confundirla con lo negativo sin más.

Mientras confundimos el (no)-ser con lo negativo, es inevitable que la contradicción sea llevada al ser; pero la contradicción es aún la apariencia o el epifenómeno, la ilusión proyectada por el problema, la sombra de una pregunta que permanece abierta y del ser que corresponde como tal a esa pregunta (antes de darle una respuesta) (1993: 89).

Si bien en la cita previa Deleuze no discute con Heidegger, sino con cierta interpretación - acaso no la más interesante- de Hegel, el pasaje sirve para mostrar que el ser problemático de lo diferencial se encuentra más acá de la contradicción como relación negativa entre elementos ya constituidos. Sin embargo, este énfasis en la sutil pero crucial distinción entre lo negativo y lo problemático muestra hasta qué punto el estatuto de lo virtual se acerca a una cierta negatividad.

Este carácter ontológico no positivo sino cuestionante o problemático de la Diferencia virtual es la manera deleuziana de sortear el impasse planteado por Pascal que ya condujera inicialmente a Heidegger a pensar la diferencia ontológica. Con claridad meridiana plantea Pascal el problema ya en el siglo XVII:

No se puede proceder a definir el ser sin caer en este absurdo: ya que no se puede definir una palabra sin comenzar por esta, es, ya sea que se la exprese o se la sobreentienda. Luego, para definir el ser, habría que decir es, y así emplear la palabra definida en su definición (Pascal, 1912: 169 [citado en Heidegger, GA 2, 5]).

Este pasaje es citado en el $\int 1$ de Sery tiempo, cuando Heidegger se refiere al carácter indefinible del ser. Luego de citar a Pascal en nota al pie, Heidegger escribe: "En efecto, el 'ser' no puede ser concebido como un ente; [...] no se puede determinar el 'ser' atribuyéndole una entidad. [...] [E]1 'ser' [Sein] no es algo así como un ente [Seiendes]" (Heidegger, 1997: 27 [GA 2, 5]). Es decir, Heidegger concluye de allí la diferencia ontológica. Ahora bien, Deleuze elige una vía distinta: en lugar de mantener la inefabilidad del ser en aras de resguardarlo de todo intento de reducción a lo ente, inventa un estatuto problemático del ser que le permite hablar de él de un modo no meramente negativo. De este modo, puede pasar de la pregunta porel ser al ser de la pregunta, del carácter problemático de la investigación ontológica a la afirmación del carácter problemático del ser en tanto tal. De cualquier manera, así como Heidegger, siguiendo el planteo pascaliano, se inclina por la expresión "hay" (es gibt) para referirse al ser (cf., e.g., Heidegger, GA 2, 304; GA 24, 13), Deleuze dirá que lo virtual no existe, sino que insiste o persiste (1993: 212). ${ }^{4}$ Se trata de dos meandros distintos para sortear el mismo problema: el de la diferencia entre lo ente y aquello que hace que lo ente sea.

Yendo, ahora sí, a la nota al pie, Deleuze busca mediante ella apoyo en Heidegger con respecto a este estatuto problemático de la diferencia - una negatividad que no es negación-: "el NO [NE-PAS] heideggeriano reenviaba, no a lo negativo en el ser, sino al ser como diferencia; y no a la negación, sino a la pregunta [question]" (1993: 89). Debemos extraer dos conclusiones de dicho pasaje: en primer lugar, que para Deleuze, no es que el ser sea nada, sino que es nada de ente, y no es que sea negativo 
en sí mismo, sino que es negatividad con respecto al ente, porque no es ente - pero en sí mismo, es diferencial y problemático-; en segundo lugar, que Deleuze atribuye estas ideas al propio Heidegger. Deleuze defiende a este respecto la concepción merleau-pontyana - de inspiración heideggeriana - del ser como pliegue (en su libro póstumo Lo visible y lo invisible) frente a los agujeros y lagos de no-ser sartrianos, y resume cinco tesis heideggerianas.

En primer lugar, escribe: "El no [ne-pas] no expresa lo negativo, sino la diferencia entre el ser y el ente" (1993: 90). Deleuze remite aquí a comentarios añadidos por Heidegger en los años '40 a dos célebres textos de 1929: "De la esencia del fundamento" y "Qué es metafísica". En cuanto al primero de estos textos, cita la segunda frase del siguiente pasaje, tomada del "Prefacio a la tercera edición (1949)":

La nada [Das Nichts] es el no [das Nicht] de lo ente y, de este modo, el ser experimentado a partir de lo ente. La diferencia ontológica es el no [das Nicht] entre ente y ser. Pero del mismo modo que el ser, en cuanto un no relativo a lo ente, no es una nada en el sentido de nihil negativum, del mismo modo, en cuanto no entre lo ente y el ser, la diferencia no es el producto de una distinción del entendimiento (ens rationis) (Heidegger, 2018: 109 [GA 9, 123]).

Dicho pasaje enfatiza el carácter negativo del ser no en tanto tal, sino con respecto a lo ente. Esto, al igual que su aprioridad con respecto a las distinciones del entendimiento, se condice plenamente con lo que sostiene el propio Deleuze. En cuanto al segundo texto, Deleuze cita un pasaje de la traducción al francés que reza como sigue: "Lo que no es nunca ni en ninguna parte un ente, ¿no se revela como el Auto-diferenciante [Se-différenciant] de todo ente?" (Heidegger, 1938: 25 [citado en Deleuze, 1993: 90]) Dicha versión es fuertemente interpretativa, puesto que, en principio, el pasaje original no parece referirse a algo que diferencia al ente, sino a aquello que se diferencia del ente. En efecto, la versión francesa de Corbin, un tanto libre, vuelca parte de la siguiente frase del "Epílogo" (1943):

Tal vez se pueda comprobar si la nada, que determina a la angustia en su esencia, se agota en una vacía negación de todo ente o si aquello que no es nunca ni en ningún lugar un ente se desvela como aquello que se diferencia [Sichunterscheidende] de todo ente y que nosotros nombramos ser. ${ }^{5}$ (Heidegger, 2018: 253 [GA 9, 305]).

Como sea, la expresión Sichunterscheidende le resulta particularmente atractiva a Deleuze, al punto de apropiarse del término y otorgarle una potencia sintética, como articulación de la diferencia que, si bien tal vez no tenga en Heidegger, la idea sí se encuentra en su obra bajo otros términos, como el de lo Mismo [das Selbe] en Identidad y diferencia - que aparecerá en el quinto punto. La expresión es inclusive retomada en alemán en el capítulo siguiente:

Es preciso que, siguiendo la intuición ontológica de Heidegger, la diferencia sea en sí misma articulación y ligazón, que relacione [rapporte] lo diferente a lo diferente, sin ninguna mediación por lo idéntico o lo semejante, lo análogo o lo opuesto. Es precisa una diferenciación de la diferencia, un en-sí como un diferenciante [différenciant], Sich-unterscheidende [sic], por la cual lo diferente

5 En el texto de Deleuze : "Ce qui n'est jamais ni nulle part un étant ne se dévoile-t-il pas comme le Se-différenciant de tout étant ?" (Citado en 1993: 90). En el original: „Es mag prüfen, ob das Nichts, das die Angst in ihr Wesen stimmt, sich bei einer leeren Verneinung alles Seienden erschöpft, oder ob, was nie und nirgends ein Seiendes ist, sich entschleiert als das von allem Seienden Sichunterscheidende, das wir das Sein nennen" (GA 9, 305). 
se encuentre al mismo tiempo reunido [rassemblé], en lugar de ser representado bajo la condición de una semejanza, de una identidad, de una analogía, de una oposición previas (1993: 154).

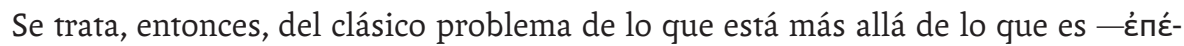

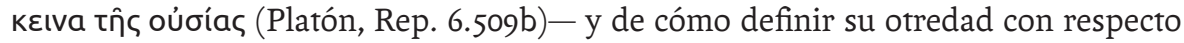
a lo ente, como queda claro en otro pasaje del Epílogo: "Eso absolutamente otro en comparación con lo ente es lo no-ente. Pero dicha nada se presenta como el ser" (2018: 253 [GA 9, 306]). Aún más reveladora es la nota que Heidegger agrega en la cuarta edición: luego de "nada" aclara "de lo ente".

Salteemos por ahora el segundo punto relevado por Deleuze para detenernos un momento en el tercero. Allí se identifica la diferencia ontológica con el estatuto ontológico propio de la pregunta, reenviando una vez más a "De la esencia del fundamento": "La diferencia ontológica corresponde a la pregunta. Es el ser de la pregunta, que se desarrolla [développe] en problemas, marcando campos determinados en relación al ente" (1993: 90). Ahora bien, esta idea no se encuentra en absoluto en dicho texto, con lo cual la hipótesis más plausible es que se trate de un desliz, y que Deleuze se esté refiriendo a otro texto. El problema con dicha hipótesis es que la idea no solo no se encuentra en "De la esencia del fundamento", sino que no se encuentra en la obra de Heidegger en general. ${ }^{6}$ A lo sumo, podríamos relacionarla algo libremente con el siguiente pasaje:

La existencia del hombre histórico comienza en ese instante en el que el primer pensador se pone al servicio del desocultamiento [Unverborgenheit] de lo ente preguntando qué sea lo ente. En esta pregunta es en donde por vez primera se experimenta el desocultamiento (Heidegger, 2018: 161 [GA 9, 189]).

Aquí tenemos, como máximo, que el preguntar da inicio a la apertura de la verdad y, como sostiene Heidegger a continuación, a la historia occidental, pero nada nos dice el pasaje sobre el serde la pregunta ni sobre su desarrollo en problemas. El único lugar de la obra heideggeriana donde encontramos algo cercano a lo que dice allí Deleuze es en el $\int 10$ de la Introducción a la investigación fenomenológica. El punto a), intitulado "La pregunta y sus estructuras", comienza como sigue:

Distinguimos en una pregunta: $1^{\circ}$ Aquello de lo que se pregunta. $2^{\circ}$ Aquello que es preguntado. $3^{\circ} \mathrm{El}$ respecto en el cual es preguntado, en relación a qué es preguntado lo que es preguntado; lo que es preguntado en ello. $4^{\circ} \mathrm{El}$ cómo del preguntar mismo, es decir, la auténtica pretensión de la respuesta. $5^{\circ} \mathrm{A}$ partir del carácter fundamental de estos momentos comprendemos la relación entre pregunta y problema. $6^{\circ}$ Cómo vienen a nuestro encuentro cosas tales como una pregunta o un problema, si preguntar es algo que yace tirado por ahí, como una piedra en el camino: el ser de la "pregunta" (Heidegger, 2008b: 85 [GA 17, 73-74]).

La enumeración de las estructuras del preguntar prosigue, pero es en los puntos $5^{\circ}$ y $6^{\circ}$ donde encontramos algo cercano a lo que Deleuze refiere, a saber, la relación entre pregunta y problema y la mención al ser de la pregunta. Sin embargo, hay que subrayar que no es una idea que se encuentre como tal en la obra de Heidegger, mucho menos si la pensamos con el peso que adquiere en la ontología deleuziana. La hipótesis del desliz, entonces, no puede sostenerse; Deleuze está haciendo algo más que interpretar: está introduciendo una tesis original de su propia ontología, donde 
Heidegger le sirve como "intercesor" (cf. Deleuze, 1990) - una práctica, por otra parte, muy cara al propio Heidegger. Es cierto, de todos modos, que la traducción francesa que leyó Deleuze se presta más a su creación. ${ }^{7}$

\subsection{Positividad}

Habiéndonos explayado sobre la cuestión de la negatividad, es preciso ahora abordar la postulada positividad deleuziana. Uno de los puntos que, a nuestro juicio, suele ser mal comprendido del filósofo francés - sin duda con apoyo textual en el juego de las superficies de Lógica del sentido y en su lectura de Spinoza a través del concepto de expresión - es pensar que se trata de una ontología de la positividad pura, del darse pleno sin sustracción. Así, por ejemplo, Hardt sostiene:

[D]eberíamos poner mucha atención desde el comienzo en distinguir la posición de Deleuze de un retorno heideggeriano a la ontología, sobre todo porque Deleuze solo aceptará respuestas 'superficiales' a la pregunta: '¿Qué hace posible al ser?' En otras palabras, nos limita a un discurso ontológico estrictamente inmanente y materialista que niega cualquier fundamento profundo u oculto del ser. El ser de Deleuze no tiene nada oculto ni negativo; está plenamente expresado en el mundo. El ser, en este sentido, es superficial, positivo y pleno (Hardt, 2005: 21-22; las itálicas son nuestras).

Independientemente del hecho de que no haya en Heidegger algo así como un "fundamento profundo u oculto del ser" - lo cual ya de por sí siembra dudas sobre la familiaridad de Hardt con el pensador alemán-, nos interesa aquí discutir esas supuestas positividad, superficialidad y plenitud del ser deleuziano. ${ }^{8}$ Ya hemos visto que la presunta "positividad" deleuziana es relativa, al igual que su supuesta plenitud y superficialidad, puesto que en Diferencia y repetición Deleuze le da una importancia fundamental a ese estatuto negativo con respecto a lo ente que es lo virtual. Ahora bien, intentaremos mostrar a continuación que no solo lo virtual ostenta un carácter no-positivo, sino que también la intensidad reviste para Deleuze una profundidad, lo cual significa que el ser no se muestra plenamente y que por tanto hay también algo así como una "negatividad" o un "ocultamiento" propio de lo envuelto intensivamente.

Si bien Deleuze no suele hablar en términos de ser y ente, su concepción de lo virtual y lo actual implica sin duda lo que Heidegger llama "diferencia ontológica". La Diferencia tiene, para Deleuze, un estatuto virtual y trascendental que se distingue de la diversidad actual y empírica, en la cual se encuentra no obstante envuelta. La intensidad es, en Diferencia y repetición, el nombre para el pliegue entre lo virtual y

7 Si comparamos la paráfrasis deleuziana con la traducción francesa utilizada, resulta evidente de dónde pudo haber sacado Deleuze dicha idea. Deleuze escribe: "La différence ontologique correspond avec l'être de la question. Elle est l'être de la question, qui se développe en problèmes, en jalonnant des champs déterminés par rapport à l'étant" (1993: 90; la itálica es nuestra). La traducción al francés de Corbin reza: "Former un concept de l'être, cela suppose que l'intelligence de l'être se soit elle-même élaborée et qu'elle ait pris expressément pour thème et pour problème l'être qui en elle est déjà compris, esquissé en un projet général et dévoilé d'une façon ou d'une autre. Entre une compréhension pré-ontologique de l'être et une problématique expressément formulée du concept de l'être, il y a de multiples degrés. Caractéristique entre autres degrés est, par exemple, le pro-jet qui, en esquissant la constitution de l'être de l'existant, jalonne en même temps un champ déterminé (Nature, Histoire) comme le domaine où il sera possible à une connaissance scientifique de constituer des objets" (Heidegger, 1938 : 57; la itálica es nuestra). Compárese con la versión original, que resulta completamente ajena a la idea que Deleuze extrae de allí: „Seinsbegreifen setzt voraus, daß das Seinsverständnis sich selbst ausgebildet und das in ihm verstandene, überhaupt entworfene und irgendwie enthüllte Sein eigens zum Thema und Problem gemacht hat. Zwischen vorontologischem Seinsverständnis und ausdrücklicher Problematik des Seinsbegreifens gibt es vielfache Stufen. Eine charakteristische ist z.B. der Entwurf der Seinsverfassung vom Seienden, durch den zugleich ein bestimmtes Feld abgesteckt wird (Natur, Geschichte) als Gebiet möglicher Vergegenständlichung durch wissenschaftliche Erkenntnis." (GA 9, Wegmarken, Vom Wesen des Grundes, 132). Como se puede notar, en este pasaje lo que hace Heidegger no es sino explicar la diferencia entre la comprensión preontológica y el concepto: "Concebir el ser presupone que se haya configurado a sí misma la propia comprensión del ser y que el ser comprendido en ella, proyectado y en cierta medida desvelado, se haya convertido propiamente en tema y problema. Entre la comprensión preontológica del ser y la problemática expresa de dicho concebir nos encontramos con múltiples grados. Un grado característico es, por ejemplo, el proyecto de la constitución del ser de lo ente mediante el cual un campo determinado (naturaleza, historia) queda delimitado simultáneamente como ámbito de una posible objetivación por parte del conocimiento científico" (2018: 116). 8 Más adelante señala también que "no deberían caber dudas de que esta concepción deleuziana de la ontología es radicalmente distinta de las concepciones hegeliana y heideggeriana, particularmente respecto de su positividad y su materialismo" (Hardt, 2005: 215). 
lo actual, lo trascendental y lo empírico o, en términos heideggerianos, el ser y el ente. Para referirse a los problemas virtuales, por su parte, Deleuze utiliza el nombre de Idea, acaso con la ambición de realizar la inversión del platonismo iniciada por Nietzsche. Esta noción de Idea no es ni la platónica - que implica una identidad del modelo y una relación de semejanza con la copia - ni la kantiana - que es condicionante pero no genética-, sino que está inspirada, entre otras cosas, en las Ideas del entendimiento de Salomon Maimon, que consisten en diferenciales que se integran en la experiencia.

Las Ideas, según Deleuze, son problemas inmanentes a sus soluciones, en las cuales persisten virtualmente. "Un problema no existe fuera de sus soluciones. Pero lejos de desaparecer, insiste y persiste en esas soluciones que lo recubren" (1993: 212). Esto significa que las Ideas no están en otro mundo, pero es precisa una determinada operación sobre el punto de vista para dar con ellas. Esta operación no depende empero de la voluntad, sino de la contingencia que un encuentro de la sensibilidad con la intensidad. La intensidad es el estado de envuelto de lo virtual en lo actual, y se distingue de la extensión, que es un estado de desenvuelto. ${ }^{9}$ En Heidegger, por su parte, este aspecto implicado propio de la intensidad en Deleuze se ve en la a $\lambda \hat{\theta} \theta \varepsilon ı$ (passim), ese movimiento a través del cual el ser se desoculta pero no sin ocultarse al mismo tiempo. En Deleuze, al ser la intensidad el ser de lo sensible, despierta a la sensibilidad a un uso no-empírico que desencadena a su vez un movimiento de todas las facultades por el cual cada una aprehende aquello que le concierne exclusivamente y que no puede captar bajo el régimen del sentido común. Es de este modo como la intensidad sacude la memoria, la imaginación, el pensamiento, trazando esa línea cortada entre los objetos trascendentes de cada facultad que no es sino la Idea como síntesis disyuntiva.

Si el orden espacial de las diferencias extrínsecas y el orden conceptual de las diferencias intrínsecas alcanzan finalmente una armonía, tal como lo prueba el esquema, ello se debe, más profundamente, a ese elemento diferencial intensivo, síntesis de lo continuo en el instante, que, bajo la forma de una continua repetitio, engendra interiormente el espacio conforme a las Ideas (1993: 40).

La intensidad, de este modo, no es solamente aquello que despierta las facultades desde el punto de vista de una teoría de la experiencia, sino también aquello que, desde el punto de vista de la lógica trascendental genética, estructura y dinamiza las Ideas mismas - de ahí que toda Idea pueda caracterizarse por los umbrales intensivos que determina. Por estas razones, no podemos pensar la intensidad sino como el pliegue o el doblez entre lo actual y lo virtual, no ya al modo de una misteriosa comunicación entre lo sensible y lo inteligible como lo es el esquematismo kantiano, sino como el sitio donde los problemas engendran soluciones y las soluciones portan problemas, es decir, el lugar de un despliegue y un repliegue ontológicos a través de los cuales el ser-problemático se desenvuelve y se extiende en entidades-soluciones que a su vez envuelven intensivamente los problemas.

Antes de retomar nuestro argumento, es preciso señalar dos consecuencias de lo anterior. En primer lugar, que hay un estatuto de lo virtual al que no le son adecuadas categorías como las de existencia o realidad efectiva, y de allí su diferencia con lo

9 Como señala Mc Namara, la noción de actual no se superpone con la de extensión; de allí que lo intensivo sea, en su lectura, actual (Mc Namara, 2020). Si bien concordamos en líneas generales con el razonamiento, creemos que no es necesario expedirse sobre el carácter actual o virtual de la intensidad, puesto que, en nuestra lectura, es aquello que nombra el pliegue entre ambos aspectos de lo real. Mc Namara tiene una razón de peso para pensar la intensidad como actual: evitar "el prejuicio platonizante que hace de lo actual una dimensión degradada" (60). La interpretación "ni (actual) ni (virtual)", no obstante, evita dicho peligro. Para un panorama de las posiciones a este respecto, cf. Clisby, 2016. 
ente. Entonces, si bien decíamos que la pareja ser/ente no suele ser propia del vocabulario deleuziano, no deja de ser cierto que enfatiza una y otra vez la "negatividad" específica del campo trascendental - tanto en su aspecto virtual como en su aspecto intensivo - en tanto no es ente. Más aún, Deleuze se refiere a esta dimensión con el término ser, si bien - como vimos- le agrega determinaciones no menores ("no" entre paréntesis, signo de pregunta). Sin embargo, al filósofo francés le resulta crucial distinguir esta "nada de ente" de la contrariedad, la oposición e incluso la mera nada, en tanto estas últimas son categorías que corresponden a lo actual. En segundo lugar, que no solo a lo virtual le es inherente una productividad, sino que la filosofía puede conceptualizar este aspecto genético. En Heidegger, por el contrario, es posible pensar una productividad del ser, pero si hay algo que queda claro a lo largo de su obra es que la lógica de dicha producción se nos escapa por completo en tanto seres finitos. El objetivo de Deleuze es mucho más ambicioso que el de Heidegger: mientras que el último toma la finitud como punto de partida, y su tarea filosófica con respecto al espacio y al tiempo es pensarlos en tanto horizontes que abren la trascendencia del Dasein, el primero se propone pensar la generación de espacio-tiempos concretos, independientemente de su ser-para alguien. No obstante, no hay que confundir esta productividad ontológica con una positividad plena.

\section{Trascendencia e inmanencia}

\subsection{Trascendencia}

Pasemos ahora a la supuesta oposición entre la trascendencia heideggeriana y la inmanencia deleuziana. Como explica sintética pero acertadamente Jean Greisch, Heidegger distingue dos figuras de la trascendencia:

la trascendencia óntica, que se confunde con la intencionalidad noética, que le permite al sujeto superarse para alcanzar un objeto, y la trascendencia ontológica, asimilada al ser-en-el-mundo que constituye él mismo 'la proto-trascendencia' (Urtranszendenz) que vuelve posible toda relación intencional referida al ente (Greisch, 2010: 81).

Esto no se opone a la inmanencia deleuziana; simplemente es otra cosa. Lo único que hay que tener allí en cuenta es que no se trata en absoluto de la trascendencia entendida en el sentido de un ente que se encuentra más allá del mundo o más allá de nuestro alcance y que actuaría como fundamento, causa u origen, ni tampoco de un sub-jectum como sustrato de representaciones. Heidegger es muy claro respecto de lo que la trascendencia no es:

La trascendencia no es una relación entre una esfera interna y una esfera externa, de tal modo que lo que sería trascendido [überschritten] allí sería un límite perteneciente al sujeto que lo separaría de la esfera externa. Pero la trascendencia no es tampoco primariamente la relación cognitiva de un sujeto con un objeto, que correspondería al sujeto como una añadidura a su subjetividad. Con más razón, la trascendencia no es simplemente el título para lo trascendente [das Überschwängliche] y lo inaccesible al conocimiento finito (GA 26, 210-211).

Lo que a Heidegger le interesa bajo el nombre "trascendencia" es la estructura mediante la cual algo en general puede ser dado o, para decirlo con Kant, las condiciones de posibilidad de la experiencia. Esto es algo de lo que ciertamente Deleuze también se ocupa, aunque no elija el término "trascendencia". Es más: en su curso conocido como ¿Qué es fundar? el propio Deleuze afirma que "con Heidegger [...] lo que desaparece es la distinción entre la trascendencia y lo trascendental" (Deleuze, 19561957) -término este último que, como se sabe, Deleuze utiliza más que gustoso-. 
Deleuze se ocupa en repetidas oportunidades a lo largo de su obra del problema que en Heidegger se llama "trascendencia": ya en Empirismo y subjetividad (1953) y La filosofía crítica de Kant (1963) da cuenta de dos explicaciones posibles acerca de cómo se constituye una experiencia; sin embargo, tanto la respuesta humeana -en la que el hábito conforma tanto un sujeto como un objeto sonsacando pequeñas diferencias a la repetición-como la kantiana - en la cual la unidad sintética de la apercepción trascendental instituye la posibilidad de referir las múltiples representaciones a una misma conciencia - son respuestas al problema de la estructura básica que posibilita la experiencia, es decir, la "trascendencia" heideggeriana. ${ }^{10}$ Desde luego, Deleuze preferirá la primera opción, puesto que allí -en contraste con la primacía del "yo pienso" kantiano- el sujeto se constituye en la experiencia con el mismo grado de originariedad que el objeto, y elaborará, a partir de su lectura de Hume, la primera síntesis del tiempo presente en el segundo capítulo de Diferencia y repetición - que es entonces, también, una respuesta al problema de la constitución de la trascendencia.

\subsection{Inmanencia}

Despejado este malentendido, habría que expedirse ahora con respecto a la inmanencia. Para ello será preciso recurrir nuevamente a la "Nota sobre la filosofía de la diferencia en Heidegger", abordando ahora el concepto de Zwiefalt (pliegue o doblez). En efecto, es allí donde podremos encontrar, en Heidegger, algo así como lo que Deleuze llama "inmanencia". De los cinco puntos relevados por Deleuze, hemos visto ya el primero y el tercero, referidos a la negatividad. Abordemos, ahora sí, el segundo, que determina la diferencia entre ser y ente como pliegue, reenviando esta vez a "Superación de la metafísica":

Esa diferencia no es "entre" en el sentido ordinario de la palabra. Ella es el Pliegue $[P(i], Z$ wiefalt. Es constitutiva del ser, y de la manera en que el ser constituye al ente, en el doble movimiento del "claro" [éclaircie] y del "encubrimiento" [voilement]. El ser es verdaderamente el diferenciante de la diferencia. De allí la expresión: diferencia ontológica. (1993: 90)

El término Zwiefalt aparece dos veces en dicho texto, aunque es el claro protagonista de "Moira", el comentario heideggeriano al Fragmento VIII, 34-41 de Parménides (1952) - texto no citado por Deleuze en Diferencia y repetición, pero sí en El pliegue (cf. Deleuze, 1988: 42). Allí, el vocablo es utilizado por Heidegger para traducir el griego ćóv. En aras de elucidar la relación entre pensar (vocîv) y ser (Eîval) nombrada en el Fragmento III ("Pues es lo mismo pensar que ser"), Heidegger acude al Fragmento VIII, donde no se habla ya de eivva sino de éóv, razón por la cual, nos dice el filósofo alemán, se suele pensar que dicho fragmento habla del ente y no del ser.

Pero Parménides, con el nombre ćóv, no piensa en modo alguno en el ente en sí [das Seiende an sich], al que, como totalidad, pertenece también el pensar, en tanto que es algo ente. Tampoco piensa el źóv como el kivvaı en el sentido del ser para sí [Seins für sich], como si al pensar le importara destacar el modo esencial no sensible del ser frente al ente como lo sensible. El ḱóv, lo que es siendo [das Seiend], está pensado más bien en el pliegue de ser y ente [Zwiefalt von Sein und Seiendem], y formulado de un modo participial [...]. El pliegue se

\footnotetext{
10 Deleuze se refiere tempranamente a la diferencia entre Hume y Kant en los siguientes términos: "Hacemos una crítica trascendental cuando, situándonos sobre un plano metódicamente reducido que nos da pues una certidumbre esencial, una certidumbre de esencia, nos preguntamos: ¿cómo puede haber lo dado, cómo algo puede darse a un sujeto, cómo el sujeto puede darse algo? Aquí, la exigencia crítica es la de una lógica constructiva que encuentra su tipo en las matemáticas. La crítica es empírica cuando, ubicándose desde un punto de vista puramente inmanente desde donde sea posible al contrario una descripción que encuentre su regla en hipótesis determinables y su modelo en física, uno se pregunta a propósito del sujeto: ¿cómo se constituye en lo dado? La construcción de este da lugar a la constitución de aquel. Lo dado ya no es dado a un sujeto, el sujeto se constituye en lo dado" (Deleuze, 1953: 92).
} 
puede por lo menos indicar por medio de los giros "ser del ente" y "ente en el ser". Ahora bien, lo que se despliega del pliegue [das Entfaltende der Zwiefalt], más que indicarnos su esencia, lo que hace es ocultarse por medio del "en el" y del "del"." (Heidegger, 1994: 208 [GA 7, 245]; traducción modificada).

Heidegger utiliza allí un término de imposible traducción, das Seiend, que no es - como lo aclara explícitamente y a diferencia de lo que traduce Barjau-el ente (das Seiende), ${ }^{12}$ sino este ćóv como pliegue o doblez entre ser y ente, mediante el cual el ser se da en el ente pero al mismo tiempo se sustrae, y el ente muestra el ser pero al mismo tiempo lo oculta. ${ }^{13}$ El desplegarse del pliegue, por el contrario, mienta el acontecimiento metafísico mediante el cual el ser es interpretado en términos entitativos, dejando caer en el olvido el aspecto sustractivo. El punto importante del concepto de pliegue es, para Deleuze, que mienta una diferencia previa a la mera distinción entre entidades ya constituidas. El pliegue entre ser del ente y ente en su ser es según Heidegger constitutivo de ambos, y en ese sentido absolutamente inapresable desde un pensar representativo que suponga que hay algo como el ser, algo como el ente, y en tercer lugar una relación entre ellos. El pliegue incluye la diferencia ontológica, como se ve aún más claramente si leemos el pasaje citado en conjunción con "De la esencia del fundamento":

La verdad óntica y la verdad ontológica conciernen ambas, de manera diferente en cada caso, a lo ente en su ser y al ser de lo ente. Ambas se pertenecen mutuamente de modo esencial, por razón de su participación en la diferencia de ser y ente (diferencia ontológica). (Heidegger, 2018: 117 [GA 9, 134]).

Esta idea de la co-pertenencia será retomada por Deleuze en el quinto punto, esta vez bajo el concepto de una Mismidad que incluye la diferencia. En cuanto al texto citado por Deleuze, "Superación de la metafísica", Heidegger se refiere allí a la Zwiefalt en términos de la historia del ser:

La metafísica es fatalidad [Verhängnis], en el sentido estricto al que aquí nos referimos, en que como rasgo fundamental de la historia occidental-europea, deja las humanidades [Menschentümer] suspendidas en medio de lo ente, sin que el ser del ente pueda jamás ser experienciado, interrogado y ensamblado en su verdad como el pliegue [Zwiefalt] de ambos, por y a través de la metafísica (Heidegger, 1994: 69 [GA 7, 76]; traducción modificada).

11 El término Zwiefaltresulta de muy difícil traducción. Contiene la raíz del término más evidente para "pliegue", Falte, pero al mismo tiempo el Zwie señala una dualidad —razón por la cual Raúl Gabás prefiere el término “duplicidad" para volcar "Zwiefalt" (cf. Heidegger, 2008: 231). Creemos no obstante que esta última elección deja escapar un matiz importante, no tanto porque la lengua alemana ya posea el término Duplizität, sino porque el concepto en cuestión apunta a "doblar" esa duplicidad en una Mismidad que contenga la diferencia. Por otra parte, el neologismo das Entfaltende es un participio presente de entfalten, desplegar, con lo cual se mantiene el juego entre el pliegue y el despliegue. Huelga decir que Deleuze jugará una y otra vez con las múltiples variantes del francés pli, que ofrece estas y otras posibilidades. 12 Como sostiene Lome Hurtado: "El pliegue es la mutua reciprocidad con la cual el ser y el ente se encuentran atados, aunque el ente es debido a que el ser está en él ya pre-comprendido, pero el ser jamás podrá ser un ente; el pliegue de ser y ente es un destino bajo el cual ambos están atados. Por ello, todas las interpretaciones que vierten la palabra 'èón' como lo 'ente' carecen de total fundamento; para Heidegger el èón no mienta lo ente, pero tampoco piensa el ser en sí mismo, sino que contiene la duplicidad, pliegue o juntura que hace comprender tanto el "'ser del ente" y "ente en el ser"' [...] bajo la expresión 'lo que es siendo, siendo lo que es"' (Lome Hurtado, 2019: 13).

13 El interés heideggeriano por el ḱóv aparece también en cursos como ¿Qué significa pensar? (1951-1952) o escritos como "La sentencia de Anaximandro" (1946). En el primero, Heidegger señala que "[l]a palabra, analizada gramaticalmente, es un participio. Nuestra reflexión ha mostrado que ćóv es el participio de todos los participios. [...] Expresa la duplicidad: lo que es siendo, siendo lo que es" (Heidegger, 2009: 231). En cuanto al segundo, el filósofo contrapone las formas arcaicas (presocráticas y homéricas) ćóv y éóvta a las posteriores őv y ővta: "En Platón y Aristóteles salen a nuestro encuentro las palabras őv y ővia como términos conceptuales. Las posteriores denominaciones 'óntico' y 'ontológico' han sido construidas sobre esa base. Sin embargo, según se supone desde el punto de vista de la lengua, őv y ővta son de algún modo la forma desgastada de las palabras originarias ćóv y źóvta. Ahora bien, en esas palabras todavía resuena eso con lo que enunciamos

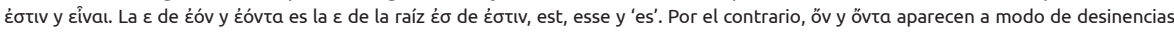
participiales y atemáticas, como si tuvieran que nombrar para sí y expresamente eso que tenemos que pensar en la forma que fue interpre-

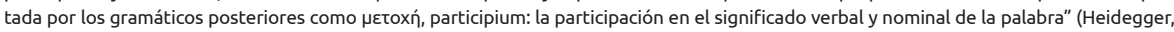
1998: 255-256 [GA 5, 317]). De este modo, marca cómo las formas arcaicas, a diferencia de las posteriores, incluyen una co-pertenencia entre aquello que realiza el verbo y el verbo mismo, es decir, incorporan la relación entre ente (őv) y ser (Eîval). Lo que expresan, pues, ćóv y ćóvta, a diferencia de őv y ővta, es el pliegue de la diferencia ontológica. 
En efecto, la metafísica no es sino la historia del olvido del pliegue y del olvido de este olvido. De allí que para Heidegger superar la metafísica equivalga, luego de su consumación con Nietzsche, a preparar el desocultamiento del doblez mediante el cual ser y ente se pliegan el uno en el otro: "Con el comienzo [Beginn] del acabamiento [Vollendung] de la metafísica comienza la preparación, desconocida y esencialmente inaccesible para la metafísica, de una primera aparición del pliegue del ser y del ente." (Heidegger, 1994: 70 [GA 7, 76]; traducción modificada.) Deleuze, hay que decirlo, siempre se mostró reacio a todas las tentativas de superación de la filosofía - precisamente, porque vio en ella la posibilidad de pensar el campo trascendental más allá de la metafísica de la presencia. ${ }^{14}$ Sin embargo, lo que Heidegger llama "metafísica" funciona del mismo modo que lo que Deleuze llama "representación": se trata en ambos casos del "olvido" de la Diferencia. Esta es una de las determinaciones características del concepto deleuziano de inmanencia: su completa ajenidad con respecto a la representación. De hecho, para Deleuze, la representación no es un concepto que se limite a la Modernidad, sino que echa raíces ya en los griegos. ${ }^{15}$ En efecto, la representación no es pensada por el filósofo francés necesariamente como relativa a un Yo, sino ante todo como una subordinación de la diferencia a la identidad. Esto puede adquirir la figura de las múltiples representaciones referidas a la unidad de la conciencia, pero su función definitoria es retrotraer siempre una multiplicidad cualquiera a la unidad de un concepto, de tal modo que el concepto de la diferencia quede velado tras la diferencia conceptual.

El cuarto punto relevado en la nota al pie sobre Heidegger va precisamente en esta línea, desligando la diferencia heideggeriana de la representación:

Así comprendida, la diferencia no es objeto de representación. La representación, como elemento de la metafísica, subordina la diferencia a la identidad, aunque más no sea relacionándola a un tertium como centro de una comparación entre dos términos que se supone que difieren (el ser y el ente) (Deleuze, 1993: 90).

Por último, el quinto punto plantea un concepto de Mismidad de la diferencia que no se reduce a la igualdad de la representación, remitiendo esta vez a Identidady diferencia, cuyo comienzo realiza precisamente esta distinción:

¿Qué dice la fórmula $\mathrm{A}=\mathrm{A}$ con la que se suele presentar el principio de identidad? La fórmula menciona la igualdad [Gleichheit] de A y A. Para una igualdad se requieren al menos dos términos. Un $A$ es igual a otro. ¿Es esto lo que quiere enunciar el principio de identidad? Evidentemente no. Lo idéntico [Identische], en latín ídem, es en griego tò aútó. Traducido a nuestra lengua alemana tò aútó quiere decir "das Selbe" [lo Mismo]. Cuando alguien dice siempre lo mismo, por ejemplo, la planta es la planta, se está expresando en una tautología. Para que algo pueda ser lo mismo, basta en cada caso un término. No precisa de un segundo término como ocurre con la igualdad ${ }^{16}$ (Heidegger, 2013: 61-63 [GA 11, 33]).

Es en este sentido que, al glosar el concepto de pliegue en Deleuze, Mengue sostiene: "Cuando Heidegger plantea lo que queda por pensar, la diferencia entre el ser y el ente, quiere orientar el pensamiento hacia una mismidad que no se relaciona en

\footnotetext{
14 "Nunca me han preocupado la superación de la metafísica o la muerte de la filosofía. La filosofía tiene una función que sigue siendo plenamente actual, crear conceptos" (Deleuze, 1990b: 186). Cf., asimismo, Deleuze y Guattari, 1991: 14; Deleuze y Parnet, 1996: 7.

15 Deleuze distingue dos figuras de la representación: la representación finita u orgánica (cuyo máximo exponente es Aristóteles) y la representación infinita u órgica (consumada por Leibniz y Hegel). Cf. Deleuze, 1993: 45-52, 61-71.

16 Dicha distinción reaparece en el segundo apartado de Identidad y diferencia, "La constitución onto-teo-lógica de la metafísica", a propósito del diálogo con el pensamiento de Hegel: "lo mismo [das Selbe] no es lo igual [das Gleiche]", ya que "[e]n lo igual desaparece la disparidad [Verschiedenheit]", mientras que "[e]n lo mismo aparece la disparidad" (2013: 105 [GA 11, 55]).
} 
nada con una identidad" (Mengue, 2008: 124). Deleuze se limita a comentar aquí que "[1]a diferencia no se deja entonces subordinar a lo Idéntico o a lo Igual, pero debe ser pensada en lo Mismo y como lo Mismo" (1993: 90). No obstante, más adelante establecerá una distinción entre tres maneras de pensar lo Mismo en relación al eterno retorno: como aquello que vuelve, como una ilusión proyectada por el funcionamiento del sistema, o como el eterno retorno mismo (cf. 1993: 164-165), quedándose con esta última opción -mencionamos este punto para marcar que lo Mismo no necesariamente es una categoría de la representación en Deleuze. En cuanto a Heidegger, posteriormente en dicho texto remitirá esta Mismidad a la mutua pertenencia entre hombre y ser, es decir, al pensamiento del Ereignis, en el cual Deleuze no se introduce. Lo importante aquí para el filósofo francés es (a) la aprioridad de la mismidad con respecto a la igualdad, y (b) que la mismidad incluye la diferencia, como se ve en la cita que introduce tomada de "...Poéticamente habita el hombre...":

Lo mismo y lo igual no se recubren más que lo mismo y la uniformidad vacía de lo puro idéntico. Lo igual se anuda siempre a lo sin-diferencia, para que todo concuerde [s'accorde] en él. Lo mismo, al contrario, es la pertenencia mutua de lo diferente a partir de la reunión [rassemblement] operada por la diferencia. No se puede decir lo mismo sino cuando la diferencia es pensada... ${ }^{17}$ Lo mismo aleja todo afán por resolver las diferencias en lo igual: por no hacer nunca otra cosa que igualar. Lo mismo reúne lo diferente en una unión original. Lo igual, al contrario, dispersa en la unidad anodina de lo uno simplemente uniforme ${ }^{18}$ (En 1993: 91).

Incluso en Identidady diferencia encontramos esta idea de la cual podemos decir sin exagerar que es la idea rectora de Diferencia y repetición: "Si intentamos representárnosla, nos encontramos inmediatamente inducidos a concebir la diferencia como una relación añadida por nuestra representación al ser y lo ente. Con ello, se rebaja la diferencia a simple distinción, a producto de nuestro entendimiento". (Heidegger, 1994: 135 [GA 11, 69]). Boundas se equivoca: el título de Diferencia y repetición no es una respuesta a Sery tiempo, sino una repetición -en el mejor de los sentidos tanto deleuziano como heideggeriano- de Identidad y diferencia. ${ }^{19}$

Los conceptos heideggerianos de Zwiefalt y Selbe, entonces, incluyen una diferencia que no se deja reducir ni a la identidad, ni a la síntesis entre entidades preexistentes. Ambos conceptos son rescatados por Deleuze precisamente por ser refractarios a la representación, en tanto que piensan un movimiento de diferenciación ontológico inmanente, previo a toda diferencia empírica. En este sentido, ambos piensan la inmanencia entre ser y ente o, como diría Heidegger, su co-pertenencia. Hay una zona de indiscernibilidad entre la diferencia ontológica heideggeriana y la inmanencia deleuziana en la medida en que ambas piensan la Relación sin mediaciones: no hay inmanencia $a$ un ente privilegiado que funcionaría como parámetro para medir la validez de todos los demás. Mejor dicho, tal cosa existe, pero Deleuze la llama "trascendencia"; Heidegger, "olvido del ser".

17 La traducción castellana agrega confusión al traducir "No puede decirse lo mismo cuando la diferencia es pensada", omitiendo el "que" francés ("sino" en castellano) que le da un sentido opuesto a la frase (cf. Deleuze, 2009: 114).

18 La traducción utilizada por Deleuze es, nuevamente, bastante poco rigurosa. Una versión más exacta en castellano podría ser la siguiente: "Lo mismo [Das selbe] no coincide nunca con lo igual [dem gleichen], tampoco con la vacía indiferencia de lo meramente idéntico [des blo $\beta$ Identischen]. Lo igual se está trasladando continuamente a lo indiferenciado [das Unterschiedlose] para que allí concuerde todo [übereinkomme]. En cambio lo mismo es la copertenencia de lo diverso por la reunión a través de la diferencia [das Zusammengehören des Verschiedenen aus der Versammlung durch den Unterschied]. Lo Mismo [Das Selbe] solo se deja decir cuando la diferencia es pensada. En el arreglo de lo diferenciado [Austrag des Unterschiedenen] adviene a la luz la esencia reunidora de lo mismo. Lo mismo proscribe todo afán de limitarse solo a igualar lo diverso [das Verschiedene] en lo igual. Lo mismo reúne lo diferente en una unión [Einigkeit] originaria. Lo igual, al contrario, disipa en la insulsa unidad [Einheit] de lo Uno [Einen] meramente uniforme" (Heidegger, 1994: 168 [GA 7, 196-197]; traducción modificada). 19 Constantin Boundas sostiene que "la elección por parte de Deleuze del título 'Diferencia y repetición' para su obra filosófica más importante era una respuesta a 'Ser y tiempo' de Heidegger", puesto que "la 'diferencia' debe eliminar los últimos vestigios de identidad en 'Ser' y el Tiempo se transforma en la Repetición del eventum tantum en el eterno retorno de la infinita diferent/ciación" (Boundas, 2009: 326-327.) 


\section{A modo de conclusión}

Desde luego, son muchas e importantes las divergencias que podrían señalarse entre Deleuze y Heidegger. El trabajo en este sentido es inagotable. La divisoria de aguas que no podemos dejar de señalar es que, mientras que el filósofo alemán mantiene al ser en una radical inefabilidad, Deleuze se propone desentrañar la lógica trascendental genética de lo virtual. Los capítulos cuarto y quinto de Diferencia y repetición están dedicados a desarrollar (1) la lógica diferencial de la determinación propia a lo ideal, que Deleuze llama diferentiación (différentiation), (2) la estructuración de lo real a través de los dinamismos espacio-temporales para los que Deleuze utiliza el término "dramatización", (3) la individuación producida por las intensidades inherentes a las diferencias entre las series que conforman las estructuras ideales, y (4) la actualización mediante la cual las singularidades ideales y sus relaciones diferenciales se disfrazan en partes y especies, respectivamente - proceso al que Deleuze llama "diferenciación" (différenciation). La unidad de dichos procesos es llamada por el autor "indi-dramadiferent/ciación" (1993: 317). Es por ello que, como sostiene Rae, "si el ser es diferencia y está continuamente diferent/ciándose [...], la filosofía no necesita ser superada para relacionarse con el ser" (Rae, 2014: 10). De allí que la práctica deleuziana de una filosofía no-representativa se distinga del pensar meditativo heideggeriano en tanto que se trata menos de recordar una ausencia que de crear conceptos adecuados al ser problemático y diferencial.

Sin embargo, puesto que la imagen de esta relación suele estar impregnada de una serie de imprecisiones, creímos necesario ante todo proceder a una clarificación de las dos principales "oposiciones" postuladas: inmanencia y trascendencia, positividad y negatividad. Creemos que estas dos aclaraciones preliminares son necesarias para cualquier ulterior investigación con respecto a la relación entre el pensamiento heideggeriano y la filosofía deleuziana. Aun así, hemos debido limitar la discusión a unos pocos núcleos problemáticos. Dado el carácter inabarcable de cada uno de estos dos pensadores, hemos optado por enfatizar aquellos conceptos que consideramos más proclives a despejar el terreno de los prejuicios más habituales. Es por ello que hemos intentado, en cuanto a la ontología deleuziana, poner de relieve, por un lado, la relativa "negatividad" de la dimensión virtual-ideal y, por el otro, el aspecto esencialmente sustractivo de la intensidad en tanto envolvente e implicante - sin desarrollar este carácter sustractivo en el pensamiento de Heidegger, carácter harto conocido y, por la misma razón, obviando el aspecto "positivo" de la ontología deleuziana. Por otra parte, hemos optado por subrayar, en Heidegger, aquellos conceptos que se acercan a la inmanencia deleuziana, expidiéndonos apenas tanto sobre la inmanencia deleuziana como sobre la trascendencia heideggeriana, por el hecho de tratarse de conceptos sobre los que ya existe un relativo consenso. Metodológicamente, hemos procurado hacer esto partiendo de los puntos centrales que Deleuze toma explícitamente de Heidegger en su obra cumbre.

Cabe mencionar, por último, que las dos oposiciones conceptuales que se han puesto en discusión en el presente trabajo tienen un punto de encuentro en el concepto deleuziano de intensidad. La intensidad funciona, en Diferencia y repetición, como el pliegue o doblez entre lo actual y lo virtual: mienta ese aspecto de lo real que no se revela y que sin embargo constituye el ser de lo que se revela. "No es un ser sensible, sino el ser de lo sensible. No es lo dado, sino aquello por lo cual lo dado es dado." (1993: 182) De este modo, suscribe por un lado al carácter sustractivo subrayado en el segundo apartado y, por el otro, a la dimensión inmanente del pliegue señalada en el tercer apartado, constituyendo de este modo, a su vez, un pliegue entre ambos aspectos. Se trata, sin lugar a dudas, de dos caminos radicalmente divergentes del pensamiento. Esperamos haber mostrado, no obstante, que estas divergencias no se encuentran donde se las suele buscar. 


\section{Bibliografía}

»Boundas, C. (2009). Heidegger. En G. Jones y J. Roffe, Deleuze's Philosophical Lineage (pp. 321-338). Edinburgh: Edinburgh University Press.

"Clisby, D. (2016). ¿El dualismo secreto de Deleuze? Versiones en disputa de la relación entre lo virtual y lo actual, trad. P. Pachilla. Ideas. Revista de filosofía moderna y contemporánea, 4, pp. 120-148.

»Deleuze, G. (1953). Empirisme et subjectivité, París, PUF.

»Deleuze, G. (1956-1957). Qu'est-ce que fonder? Curso de hypokhâgne, Lycée Louis le Grand. Recuperado de https://www.webdeleuze.com/textes/218 (sin paginación).

»Deleuze, G. (1969). Logique du sens. París: Minuit.

》Deleuze, G. (1986). Foucault. París: Minuit.

"Deleuze, G. (1988). Le Pli. Leibniz et le baroque. París: Minuit.

»Deleuze, G. (1988b). Un philosophe nomade. Magazine littéraire, 257.

"Deleuze, G. (1990). Les intercesseurs. En Pourparlers (pp. 163-164). París: Minuit.

"Deleuze, G. (1990b). Sur la philosophie. En Pourparlers (pp. 185-212). París: Minuit.

»Deleuze, G. (1992). Un précurseur méconnu de Heidegger, Alfred Jarry. En Critique et clinique (pp. 115-125). París: Minuit.

»Deleuze, G. (1993 [1968]). Différence et répétition. París: PUF.

»Deleuze, G. (2002). En créant la pataphysique, Jarry a ouvert la voie à la phénoménologie. L'île déserte et autres textes. Textes et entretiens (1953-1974) (pp. 105108). París: Minuit.

»Deleuze, G. (2009). Diferencia y repetición, trad. Silvia Delpy y Hugo Beccacece. Buenos Aires: Amorrortu.

»Deleuze, G. y Guattari, F. (1991). Qu'est-ce que la philosophie? París: Minuit.

»Deleuze, G. y Parnet, C. (1996). Dialogues. París: Flammarion.

"Dosse, F. (2009). Gilles Deleuze y Félix Guattari. Biografía cruzada, trad. Sandra Garzonio. Buenos Aires: Fondo de Cultura Económica.

"Greisch, J. (2010). La invención de la diferencia ontológica, trad. Julián Fava. Buenos Aires: Las cuarenta.

" Hardt, M. (2005). Deleuze. Un aprendizaje filosófico, trad. Alcira Bixio. Buenos Aires: Paidós.

» Heidegger, M. (1938). Qu'est-ce que la métaphysique? Suivi d'extraits sur l'être et le temps et d'une conférence sur Hölderlin, trad. Henry Corbin. París: Gallimard.

"Heidegger, M. (1975). Die Grundprobleme der Metaphysik (Gesamtausgabe. II. Abteilung: Vorlesungen 1923-1944 - Band 24). Frankfurt am Main: Vittorio Klostermann.

》Heidegger, M. (1976). Wegmarken (Gesamtausgabe. I. Abteilung: Veröffentlichte Schriften 1910-1976 — Band 9). Frankfurt am Main: Vittorio Klostermann.

» Heidegger, M. (1977 [1927]). Sein und Zeit (Gesamtausgabe. I. Abteilung: Veröffent- 
lichte Schriften 1910-1976 — Band 2). Frankfurt am Main: Vittorio Klostermann.

»Heidegger, M. (1977b). Holzwege (Gesamtausgabe. I. Abteilung: Veröffentlichte Schriften 1910-1976 — Band 5). Frankfurt am Main: Vittorio Klostermann.

» Heidegger, M. (1978). Metaphysische Anfangsgründe der Logik im Ausgang von Leibniz (Gesamtausgabe. II. Abteilung: Vorlesungen 1923-1944 - Band 26). Frankfurt am Main: Vittorio Klostermann.

" Heidegger, M. (1994). Conferencias y artículos, trad. Eustaquio Barjau. Barcelona: Ediciones del Serbal.

"Heidegger, M. (1997). Ser y tiempo, trad. Jorge Eduardo Rivera. Santiago de Chile: Editorial Universitaria.

» Heidegger, M. (1998). Caminos de bosque, trad. Helena Cortés y Arturo Leyte. Madrid: Alianza.

» Heidegger, M. (2000). Vorträge und Aufsätze (Gesamtausgabe. I. Abteilung: Veröffentlichte Schriften 1910-1976 — Band 7). Frankfurt am Main: Vittorio Klostermann.

» Heidegger, M. (2006). Identität und Differenz (Gesamtausgabe. I. Abteilung: Veröffentlichte Schriften 1910-1976 - Band 7). Frankfurt am Main: Vittorio Klostermann.

» Heidegger, M. (2007 [1951]). El ser y el tiempo, trad. José Gaos. Buenos Aires: Fondo de Cultura Económica.

» Heidegger, M. (2008). ¿Qué significa pensar?, trad. Raúl Gabás. Madrid: Trotta.

"Heidegger, M. (2008b). Introducción a la investigación fenomenológica, trad. Juan José García Norro. Madrid: Síntesis.

» Heidegger, M. (2013). Identidad y diferencia. Identität und Differenz, ed. bilingüe, trad. Helena Cortés y Arturo Leyte. Madrid: Ánthropos.

» Heidegger, M. (2018). Hitos, trad. Helena Cortés y Arturo Leyte. Madrid: Alianza.

» Lome Hurtado, L. A. (2019), Martin Heidegger y el participio de todos los participios. Aporía. Revista Internacional de Investigaciones Filosóficas, 17, pp. 4-21.

» López, F. (2020). Pensamiento. En M. Soich y J. Ferreyra (eds.), Introducción en Diferencia y repetición (pp. 147-159). Buenos Aires: RAGIF.

» Mc Namara, R. (2020). Intensidad. En M. Soich y J. Ferreyra (eds.), Introducción en Diferencia y repetición (pp. 49-61). Buenos Aires: RAGIF.

" Mengue, P. (2008). Deleuze o el sistema de lo múltiple, trad. Julián Fava y Luciana Tixi. Buenos Aires: Las cuarenta.

"Pascal, B. (1912). Pensées et Opuscules. París: Brunschvicg.

» Rae, G. (2014). Ontology in Heidegger and Deleuze: A Comparative Analysis. Londres: Palgrave Macmillan.

»van Tuinen, S. y McDonnell, N. (2010). Deleuze and The Fold: A Critical Reader. Londres: Palgrave Macmillan. 